\title{
Cluster sum rules for three-body systems with angular-momentum dependent interactions
}

\author{
R. de Diego and E. Garrido \\ Instituto de Estructura de la Materia, CSIC, Serrano 123, E-28006 Madrid, Spain
}

A. S. Jensen and D. V. Fedorov

Department of Physics and Astronomy, University of Aarhus, DK-8000 Aarhus C, Denmark

(Received 31 August 2007; revised manuscript received 18 December 2007; published 5 February 2008)

\begin{abstract}
We derive general expressions for non-energy-weighted and energy-weighted cluster sum rules for systems of three charged particles. The interferences between pairs of particles are found to play a substantial role. The energy-weighted sum rule is usually determined by the kinetic energy operator, but we demonstrate that it has similar additional contributions from the angular momentum and parity dependence of two- and three-body potentials frequently used in three-body calculations. The importance of the different contributions is illustrated with the dipole excitations in ${ }^{6} \mathrm{He}$. The results are compared with the available experimental data.
\end{abstract}

DOI: 10.1103/PhysRevC.77.024001

PACS number(s): 21.45.-v, 11.55.Hx, 23.20.-g, 31.15.xj

\section{MOTIVATION}

The use of sum rules in quantum mechanics is well established and abundantly applied for many different systems $[1,2]$. The prominent examples are the transitions from a given quantum state induced by an electromagnetic multipole operator. For any multipole operator acting on an initial state, the sum of all the related transition probabilities multiplied by powers of the excitation energy are completely determined by the properties of the initial state [3,4].

The sum rules exist in general for any many-body quantum system. Of specific interest are those systems where the constituents clusterize, such that the degrees of freedom can be divided into the internal ones corresponding to each cluster and those associated with the relative motion of the clusters [5]. Then the different multipole operators can be decomposed into terms depending on the intrinsic coordinates of each cluster and an additional term depending only on the relative coordinates of the centers of mass of the clusters. This operator structure then leaves two sum rules showing the same decomposition: the sum rules associated with each individual cluster (depending only on the properties of the initial cluster state) plus the cluster sum rule (depending on the properties of the few-body initial wave function). Examples are found in Refs. [6,7], where the dipole non-energy-weighted and dipole energy-weighted sum rules are obtained for many-body systems clusterizing into a two-body system.

When a clusterized system can be properly described as a few-body system where the internal cluster degrees of freedom are frozen, only the cluster sum rules remain, corresponding to the much smaller Hilbert space of ground and excited states of the relative cluster motion. This kind of few-body descriptions have been extensively used in nuclear physics during the past $10-15$ years in connection with halos and weakly bound states in general [5]. The most interesting and frequently investigated of these systems are approximated by a three-body structure. Extensions to excited three-body continuum states are now being pursued and attracting a lot of attention [8-12]. To get accurate three-body wave functions the Faddeev decomposition with different Jacobi coordinates is employed in coordinate space computations [13]. The unavoidable transformation from one set of Jacobi coordinates to another complicates the structure of the cluster sum rules, especially when more than one of the three particles is charged.

The purpose of this work is to generalize the dipole twobody cluster sum rule as in Refs. [6,7] to three-body systems for any multipolarity. Advanced three-body calculations employ partial-wave-dependent pair interactions and state-dependent three-body potentials, and these complications must therefore also be taken into account in derivations of the cluster sum rules. Typically, the two-body interactions are adjusted independently for each partial wave to reproduce the available properties of the corresponding two-body system (e.g., bound state and resonance energies and phase shifts) $[14,15]$. These interactions are then essentially nonlocal through their angular momentum dependence. Also, it is common to introduce effective angular momentum and parity-dependent three-body forces for fine-tuning the crucial total energies of the threebody states. Since the multipole transition operators carry both angular momentum and parity they do not commute, in general, with the angular-momentum-dependent two- and three-body potentials. Thus, the energy-weighted cluster sum rule should then be rederived by including the corresponding contributions in addition to the usual kinetic energy term. In all cases we must include contributions from the three Faddeev components, which are expressed in their respective Jacobi coordinates.

In Sec. II we briefly introduce the coordinates used and summarize some important relations and definitions. In Sec. III we derive the non-energy-weighted sum rule. The energyweighted sum rule is obtained in Sec. IV, which is divided into three subsections corresponding to the contributions from the kinetic energy operator, the partial-wave-dependent two-body potentials, and the (total angular momentum) $J^{\pi}$-dependent three-body forces. As an illustration, in Sec. V we investigate the dipole excitations in ${ }^{6} \mathrm{He}$ and compare with the available experimental data. We close the paper with a short summary and the conclusions. A few intermediate expressions obtained in the derivations have been collected in the Appendix. 


\section{THE TRANSITION PROBABILITY}

We assume three clusters with masses $m_{i}$ and charges $z_{i}(i=1,2,3)$, described by coordinates $\boldsymbol{r}_{i}$, and with the three-body center of mass at $\boldsymbol{R}$. The three sets of mass-scaled Jacobi coordinates are $\left\{\boldsymbol{x}_{i}, \boldsymbol{y}_{i}\right\} \equiv\left\{\rho, \alpha_{i}, \Omega_{x_{i}}, \Omega_{y_{i}}\right\}$, where $\rho$ is the hyperradius and $\left\{\alpha_{i}, \Omega_{x_{i}}, \Omega_{y_{i}}\right\}$ are the five hyperangles corresponding to the Jacobi set $i$ (see, e.g., Ref. [13]). The connection between the Cartesian and the mass-scaled Jacobi coordinates is given by

$$
\begin{aligned}
\boldsymbol{r}_{j}-\boldsymbol{r}_{k} & =\sqrt{\frac{m_{N}\left(m_{j}+m_{k}\right)}{m_{j} m_{k}}} \boldsymbol{x}_{i}, \\
\boldsymbol{r}_{i}-\boldsymbol{R} & =\sqrt{\frac{m_{N}}{m_{i}} \frac{\left(m_{j}+m_{k}\right)}{M}} \boldsymbol{y}_{i},
\end{aligned}
$$

where $M=m_{i}+m_{j}+m_{k}$ and $m_{N}$ is the normalization mass. The transformations between different sets of Jacobi coordinates are given by

$$
\begin{aligned}
\boldsymbol{y}_{i} & =-\boldsymbol{x}_{k} \sin \varphi_{i k}-\boldsymbol{y}_{k} \cos \varphi_{i k}, \\
\tan \varphi_{i k} & =(-1)^{p} \sqrt{\frac{m_{j} M}{m_{i} m_{k}}}(\text { if } i \neq k), \quad \text { and } \quad \varphi_{i i}=\pi,
\end{aligned}
$$

which formally amounts to a rotation depending on the mass ratios and the parity $(-1)^{p}$ of the permutation $p$ of $\{i, j, k\}$. Equations (3) and (4) lead to an important relation between harmonic polynomials in different Jacobi coordinates, that is,

$$
\begin{aligned}
y_{i}^{\lambda} Y_{\lambda, \mu}\left(\Omega_{y_{i}}\right)= & \sum_{\ell=0}^{\lambda}(-1)^{\lambda} x_{k}^{\lambda-\ell}\left(\sin \varphi_{i k}\right)^{\lambda-\ell} y_{k}^{\ell}\left(\cos \varphi_{i k}\right)^{\ell} \\
& \times \sqrt{\frac{4 \pi(2 \lambda+1) !}{(2 \ell+1) !(2 \lambda-2 \ell+1) !}} \\
& \times\left[Y_{\lambda-\ell}\left(\Omega_{x_{k}}\right) \otimes Y_{\ell}\left(\Omega_{y_{k}}\right)\right]^{\lambda \mu}
\end{aligned}
$$

Let us consider the initial three-body state $\left|n_{0} J_{0} M_{0}\right\rangle$, where $J_{0}$ is the total angular momentum with projection $M_{0}$. All the other needed quantum numbers are collected into $n_{0}$. The excited states $\{|n J M\rangle\}$ can be populated from the ground state by the electric multipole operator

$$
O_{\mu}^{\lambda}=\sum_{i=1}^{3} z_{i}\left|\boldsymbol{r}_{i}-\boldsymbol{R}\right|^{\lambda} Y_{\lambda, \mu}\left(\Omega_{y_{i}}\right)
$$

where $i$ runs over the three clusters, or equivalently, over the three sets of Jacobi coordinates.

The transition probability corresponding to this electric multipole operator is proportional to the $\mathcal{B}(E \lambda)$-value, that is,

$$
\mathcal{B}\left(E \lambda, n_{0} J_{0} \rightarrow n J\right)=\sum_{\mu M}\left|\left\langle n J M\left|O_{\mu}^{\lambda}\right| n_{0} J_{0} M_{0}\right\rangle\right|^{2},
$$

from which the $\lambda$-multipole strengths are defined as

$$
S_{m}=\sum_{n J}\left(E_{n J}-E_{0}\right)^{m} \mathcal{B}\left(E \lambda, n_{0} J_{0} \rightarrow n J\right),
$$

where $E_{0}$ is the energy of the initial state, and $E_{n J}$ is the energy of the excited state with angular momentum $J$ and additional quantum numbers $n$.

The values of these multipole strengths, depending only on the properties of the initial state, are known as the sum rules. In this work we are concentrating on the sum rules with $m=0,1$, also denoted as non-energy-weighted and energyweighted sum rules, respectively.

For the dipole case $(\lambda=1)$, after inclusion of Eq. (5) into (6), and using Eqs. (2) and (4), one can see that for three particles having equal value of the ratio $z_{i} / m_{i}$ the dipole operator is zero. This means that for the particular case of three identical particles all the dipole strengths $S_{m}$ in Eq. (8) are zero.

\section{THE NON-ENERGY-WEIGHTED SUM RULE}

The sum over all transitions can be rewritten provided the intermediate set of quantum numbers gives a complete description of the (bound and continuum) final states, that is, $\sum_{n J M}|n J M\rangle\langle n J M|=1$. We then get

$$
\begin{aligned}
S_{0} & =\sum_{n J} \mathcal{B}\left(E \lambda, n_{0} J_{0} \rightarrow n J\right) \\
& =\sum_{n J} \sum_{\mu M}\left\langle n_{0} J_{0} M_{0}\left|O_{\mu}^{\lambda \dagger}\right| n J M\right\rangle\left\langle n J M\left|O_{\mu}^{\lambda}\right| n_{0} J_{0} M_{0}\right\rangle \\
& =\sum_{\mu}\left\langle n_{0} J_{0} M_{0}\left|O_{\mu}^{\lambda \dagger} O_{\mu}^{\lambda}\right| n_{0} J_{0} M_{0}\right\rangle,
\end{aligned}
$$

which for a given multipole operator is entirely determined by the properties of the ground state $\left|n_{0} J_{0} M_{0}\right\rangle$.

The definition in Eq. (6), together with Eqs. (2) and (5), permits expressing the operator $O_{\mu}^{\lambda \dagger} O_{\mu}^{\lambda}$ in terms of a single set of Jacobi coordinates, leading to

$$
\begin{aligned}
S_{0}= & \sum_{i, k=1}^{3} \sum_{\ell=0}^{\lambda} \sum_{\mu=-\lambda}^{\lambda}(-1)^{\mu} z_{i} z_{k} \sqrt{\frac{4 \pi(2 \lambda+1) !(2 \lambda+1)}{(2 \ell+1) !(2 \lambda-2 \ell+1) !}} \\
& \times\left(c_{i}\right)^{\lambda}\left(c_{k}\right)^{\lambda}\left(\sin \varphi_{i k}\right)^{\lambda-\ell}\left(\cos \varphi_{i k}\right)^{\ell} \sum_{m_{1} m_{2}}\left(\begin{array}{ccc}
\ell & \lambda-\ell & \lambda \\
m_{1} & m_{2} & -\mu
\end{array}\right) \\
& \times\left\langle n_{0} J_{0} M_{0}\right| x_{k}^{\lambda-\ell} y_{k}^{\lambda+\ell} Y_{\lambda, \mu}^{*}\left(\Omega_{y_{k}}\right) Y_{\ell, m_{1}}\left(\Omega_{y_{k}}\right) \\
& \times Y_{\lambda-\ell, m_{2}}\left(\Omega_{x_{k}}\right)\left|n_{0} J_{0} M_{0}\right\rangle,
\end{aligned}
$$

where the constants

$$
c_{i}=\sqrt{\frac{m_{N}}{m_{i}} \frac{\left(m_{j}+m_{k}\right)}{M}}
$$

arise when inserting Eq. (2) into the definition in Eq. (6).

The summation over the indexes $\mu$ and $m_{1}$ can be made analytically [16], leading to the final expression for the nonenergy-weighted sum rule:

$$
\begin{aligned}
S_{0}= & \sum_{i, k=1}^{3} \sum_{\ell=0}^{\lambda} z_{i} z_{k}\left(\begin{array}{ccc}
\lambda & \lambda-\ell & \ell \\
0 & 0 & 0
\end{array}\right) \frac{2 \lambda+1}{\sqrt{2(\lambda-\ell)+1}} \\
& \times\left(c_{i}\right)^{\lambda}\left(c_{k}\right)^{\lambda} \sqrt{\frac{(2 \lambda+1) !}{(2 \ell) !(2 \lambda-2 \ell+1) !}}\left(\sin \varphi_{i k}\right)^{\lambda-\ell}\left(\cos \varphi_{i k}\right)^{\ell}
\end{aligned}
$$




$$
\begin{aligned}
& \times \sum_{m_{2}}\left\langle n_{0} J_{0} M_{0}\right| x_{k}^{\lambda-\ell} y_{k}^{\lambda+\ell} Y_{\lambda-\ell, m_{2}}\left(\Omega_{x_{k}}\right) \\
& \times Y_{\lambda-\ell, m_{2}}^{*}\left(\Omega_{y_{k}}\right)\left|n_{0} J_{0} M_{0}\right\rangle .
\end{aligned}
$$

The sum $\left(S_{0}\right)_{\text {diag }}$ of the diagonal terms $(i=k)$ in Eq. (12) is obtained by taking $\varphi_{i i}=\pi$ and $\ell=\lambda$ [which reduce Eqs. (3) and (5) to identities], which leads to

$$
\left(S_{0}\right)_{\mathrm{diag}}=\frac{2 \lambda+1}{4 \pi} \sum_{i=1}^{3} z_{i}^{2}\left\langle J_{0} M_{0}|| \boldsymbol{r}_{i}-\left.\boldsymbol{R}\right|^{2 \lambda} \mid J_{0} M_{0}\right\rangle .
$$

For a three-body system containing only one charged particle the non-energy-weighted sum rule reduces to one of the three diagonal terms in Eq. (13). When more than one charged particle enters in the three-body system, the full expression [Eq. (12)], which contains interferences between charged particles, must be used.

The relevance of the nondiagonal terms can be easily seen for a system containing three identical particles with mass $m$ and charge $z$ for $\lambda=1$. In this case the sum of the diagonal contributions $3 \frac{3 z^{2}}{4 \pi}\left\langle\left|\boldsymbol{r}_{p}-\boldsymbol{R}\right|^{2}\right\rangle$ given by Eq. (13) is fully canceled by the nondiagonal terms, such that $S_{0}=0$, as expected for three identical particles.

When only two of the particles with mass $m$ each have the charge $z$, one of the diagonal terms is canceled out by the nondiagonal one, and we get $S_{0}=\frac{3 z^{2}}{4 \pi}\left\langle\left|\boldsymbol{r}_{p}-\boldsymbol{R}\right|^{2}\right\rangle$, which is identical to the result when only one particle is charged. These results are summarized in the second column in Table I.

\section{THE ENERGY-WEIGHTED SUM RULE}

The energy-weighted sum rule is most easily obtained by evaluating the expectation value of the double commutator in the ground state, that is,

$$
\begin{aligned}
S_{1} & =\frac{1}{2} \sum_{\mu}\left\langle n_{0} J_{0} M_{0}\left|\left[\left[O_{\mu}^{\lambda \dagger}, H\right], O_{\mu}^{\lambda}\right]\right| n_{0} J_{0} M_{0}\right\rangle \\
& =\sum_{\mu}\left\langle n_{0} J_{0} M_{0}\left|O_{\mu}^{\lambda \dagger} H O_{\mu}^{\lambda}-E_{0} O_{\mu}^{\lambda \dagger} O_{\mu}^{\lambda}\right| n_{0} J_{0} M_{0}\right\rangle,
\end{aligned}
$$

where $E_{0}$ is the ground-state energy.

This expression is obtained by inserting the identity operator $1=\sum_{n J M}|n J M\rangle\langle n J M|$ between $H$ and $O_{\mu}^{\lambda}$ and between

TABLE I. Non-energy-weighted sum rule $\left(S_{0}\right)$ and the contribution of the kinetic energy operator to the energy-weighted sum rule $\left(S_{1}^{(T)}\right)$ for a system of three particles with equal mass $(m)$ for $\lambda=1$. The first column gives the number $N$ of charged particles each with charge $z$. The symbol \langle\rangle denotes expectation value in the initial state, and $\boldsymbol{r}_{p}$ is the coordinate for one of the charged particles.

\begin{tabular}{ccc}
\hline \hline$N$ & $S_{0}$ & $S_{1}^{(T)}$ \\
\hline 1 & $3 z^{2}\left\langle\left|\boldsymbol{r}_{p}-\boldsymbol{R}\right|^{2}\right\rangle /(4 \pi)$ & $3 \hbar^{2} z^{2} /(4 \pi m)$ \\
2 & $3 z^{2}\left\langle\left|\boldsymbol{r}_{p}-\boldsymbol{R}\right|^{2}\right\rangle /(4 \pi)$ & $3 \hbar^{2} z^{2} /(4 \pi m)$ \\
3 & 0 & 0 \\
\hline \hline
\end{tabular}

$O_{\mu}^{\lambda \dagger}$ and $O_{\mu}^{\lambda}$, where $\{|n J M\rangle\}$ are the complete set of eigenstates of $H$ with the corresponding set of eigenvalues $\left\{E_{n J}\right\}$. In this way we immediately recover the standard definition in Eq. (8):

$$
S_{1}=\sum_{n J}\left(E_{n J}-E_{0}\right) \sum_{\mu M}\left|\left\langle n J M\left|O_{\mu}^{\lambda}\right| n_{0} J_{0} M_{0}\right\rangle\right|^{2} .
$$

According to Eq. (14) the energy-weighted sum rule depends on the multipole operator, the initial state properties, and the Hamiltonian. This Hamiltonian can have a complicated angular momentum dependence of both two- and three-body interactions, whose contributions to $S_{1}$ in general do not vanish. In particular we shall assume two-body interactions that depend on the relative partial wave between the two particles and three-body potentials depending on the total angular momentum and parity of the three-body state.

In the following we evaluate the expression (14) separately for the different terms of the Hamiltonian, that is, the traditional contribution from the kinetic energy operator and the new terms arising from the partial-wave-dependent two-body potentials and $J^{\pi}$-dependent three-body potentials.

\section{A. Kinetic Energy Operator}

The kinetic energy operator can be expressed in terms of any of the three sets of Jacobi coordinates as

$$
T=-\left(\Delta_{x_{k}}+\Delta_{y_{k}}\right) \hbar^{2} / 2 m_{N},
$$

where the two Laplace operators $\Delta_{x_{k}}$ and $\Delta_{y_{k}}$ are associated to the Jacobi coordinates $\boldsymbol{x}_{k}$ and $\boldsymbol{y}_{k}$. Since the multipole operator [Eq. (6)] only depends on $\boldsymbol{y}$-coordinates we can quickly find the commutator $\left[O_{\mu}^{\lambda \dagger}, T\right]$ [Eq. (A1)], from which we get

$$
\begin{aligned}
{\left[\left[O_{\mu}^{\lambda \dagger}, T\right], O_{\mu}^{\lambda}\right]=} & \sum_{i, k=1}^{3} z_{i} z_{k} \frac{\hbar^{2}}{m_{N}} \nabla_{y_{k}}\left[\left|\boldsymbol{r}_{k}-\boldsymbol{R}\right|^{\lambda} Y_{\lambda, \mu}^{*}\left(\Omega_{y_{k}}\right)\right] \\
& \cdot \nabla_{y_{k}}\left[\left|\boldsymbol{r}_{i}-\boldsymbol{R}\right|^{\lambda} Y_{\lambda, \mu}\left(\Omega_{y_{i}}\right)\right]
\end{aligned}
$$

Equation (5) permits rewriting of Eq. (17) in terms of a single set of Jacobi coordinates, leading to

$$
\begin{aligned}
& {\left[\left[O_{\mu}^{\lambda \dagger}, T\right], O_{\mu}^{\lambda}\right]=\frac{\hbar^{2}}{m_{N}} \sum_{i, k=1}^{3}\left(c_{i}\right)^{\lambda}\left(c_{k}\right)^{\lambda} z_{i} z_{k} \sum_{\ell=0}^{\lambda} \sum_{m n}(-1)^{-\mu}} \\
& \times \sqrt{2 \lambda+1}\left(\begin{array}{ccc}
\ell & \lambda-\ell & \lambda \\
m & n & -\mu
\end{array}\right) \sqrt{\frac{4 \pi(2 \lambda+1) !}{(2 \ell+1) !(2 \lambda-2 \ell+1) !}} \\
& \times x_{k}^{\lambda-\ell} Y_{\lambda-\ell, n}\left(\Omega_{x_{k}}\right)\left(\sin \varphi_{i k}\right)^{\lambda-\ell}\left(\cos \varphi_{i k}\right)^{\ell} \\
& \times \nabla_{y_{k}}\left[y_{k}^{\lambda} Y_{\lambda, \mu}^{*}\left(\Omega_{y_{k}}\right)\right] \cdot \nabla_{y_{k}}\left[y_{k}^{\ell} Y_{\ell, m}\left(\Omega_{y_{k}}\right)\right] .
\end{aligned}
$$

The scalar product can now be performed by use of the gradient formula (A2), and after writing the two spherical harmonics in terms of a single one, and performing analytically the summations over angular momentum projection quantum numbers (details of which are given in the Appendix), one gets the following final expression for the contribution of the kinetic energy operator to the energy-weighted sum 
rule $S_{1}^{(T)}$ :

$$
\begin{aligned}
& S_{1}^{(T)}=\frac{-\hbar^{2}}{2 m_{N}} \sum_{i, k=1}^{3}\left(c_{i}\right)^{\lambda}\left(c_{k}\right)^{\lambda} z_{i} z_{k} \sum_{\ell=1}^{\lambda}\left(\sin \varphi_{i k}\right)^{\lambda-\ell}\left(\cos \varphi_{i k}\right)^{\ell} \\
& \times \sqrt{\frac{\lambda \ell(2 \ell-1)(2 \lambda+1) !(2 \lambda+1)^{3}}{(2 \ell) !(2 \lambda-2 \ell+1)(2 \lambda-2 \ell+1) !}} \\
& \times\left(\begin{array}{ccc}
\lambda-1 & \ell-1 & \lambda-\ell \\
0 & 0 & 0
\end{array}\right) \\
& \times \sum_{n}\left\langle n_{0} J_{0} M_{0}\right| x_{k}^{\lambda-\ell} y_{k}^{\lambda+\ell-2} Y_{\lambda-\ell, n}\left(\Omega_{x_{k}}\right) \\
& \times Y_{\lambda-\ell, n}^{*}\left(\Omega_{y_{k}}\right)\left|n_{0} J_{0} M_{0}\right\rangle,
\end{aligned}
$$

where the sum of the diagonal parts $(i=k)$ becomes

$$
\begin{aligned}
\left(S_{1}^{(T)}\right)_{\text {diag }}= & \frac{\hbar^{2}}{2 m_{N}} \frac{\lambda(2 \lambda+1)^{2}}{4 \pi} \sum_{i=1}^{3}\left(c_{i}\right)^{2} z_{i}^{2}\left\langle n_{0} J_{0} M_{0}\right| \\
& \times\left|\boldsymbol{r}_{i}-\boldsymbol{R}\right|^{2 \lambda-2}\left|n_{0} J_{0} M_{0}\right\rangle,
\end{aligned}
$$

where the constants $c_{i}$ are given by Eq. (11).

For $\lambda=1$ the expression in Eq. (19) is independent of the properties of the initial state. In particular, for three identical particles with mass $m$ and charge $z$ the total value of $S_{1}^{(T)}$ is zero, which confirms the result anticipated at the end of Sec. II. When one of these three particles has no charge, $S_{1}^{(T)}$ takes a constant value, which is the same as the one obtained when only one of the three particles with mass $m$ is charged. The precise expressions of $S_{1}^{(T)}$ for these particular cases are given in the last column of Table I.

\section{B. Partial-Wave-Dependent Two-Body Potentials}

Typically, the two-body interactions are adjusted separately for the individual partial waves to reproduce the known experimental data for the two-body systems. This procedure leads to two-body interactions depending on the two-body quantum numbers $\left\{\ell_{x}, s_{x}, j_{x}\right\}$. The full two-body potential operator takes the form $\hat{V}_{2 b}=\sum_{i=1}^{3} \hat{V}_{2 b}^{(i)}$, where the index $i$ runs over all the three sets of Jacobi coordinates and $\hat{V}_{2 b}^{(i)}$ is the two-body operator describing the interaction between particles $j$ and $k$. This two-body operator is formally written as

$$
\begin{aligned}
\hat{V}_{2 b}^{(i)}= & \sum_{\ell_{x_{i}}, s_{x_{i}}} \sum_{j_{x_{i}}, m_{x_{i}}} V_{i}^{\left(\ell_{x_{i}}, s_{x_{i}}, j_{x_{i}}\right)}\left(x_{i}\right) \hat{P}_{i} \\
& \times\left|\ell_{x_{i}}, s_{x_{i}}, j_{x_{i}}, m_{x_{i}}\right\rangle\left\langle\ell_{x_{i}}, s_{x_{i}}, j_{x_{i}}, m_{x_{i}}\right|,
\end{aligned}
$$

where $\hat{P}_{i}$ represents any spin operator that could enter in the two-body potentials.

The contribution of the full two-body potential operator $\hat{V}_{2 b}$ to the second sum rule has then three contributions, each corresponding to one of the three two-body interactions. According to Eq. (14), the contribution $S_{1}^{(2 b, i)}(i=1,2,3)$ from each of them is given by

$$
\begin{aligned}
S_{1}^{(2 b, i)}= & \sum_{\mu}\left[\left\langle n_{0} J_{0} M_{0}\left|O_{\mu}^{\lambda \dagger} \hat{V}_{2 b}^{(i)} O_{\mu}^{\lambda}\right| n_{0} J_{0} M_{0}\right\rangle\right. \\
& \left.-\left\langle n_{0} J_{0} M_{0}\left|O_{\mu}^{\lambda \dagger} O_{\mu}^{\lambda} \hat{V}_{2 b}^{(i)}\right| n_{0} J_{0} M_{0}\right\rangle\right],
\end{aligned}
$$

where $\left|n_{0} J_{0} M_{0}\right\rangle$ represents the initial state with total angular momentum $J_{0}$ and projection $M_{0}$. The quantum number $n_{0}$ refers to all other additional quantum numbers necessary to specify this state.

For each two-body interaction $\hat{V}_{2 b}^{(i)}$ it is now convenient to write the corresponding ground-state wave function $\Psi$ in terms of the Jacobi coordinates $\left\{\boldsymbol{x}_{i}, \boldsymbol{y}_{i}\right\} \equiv\left\{\rho, \alpha_{i}, \Omega_{x_{i}}, \Omega_{y_{i}}\right\}$ and expand it in terms of a set of functions $\mathcal{Y}_{\gamma_{i}}^{J_{0} M_{0}}\left(\Omega_{i}\right)$ :

$$
\Psi_{n_{0}}^{J_{0} M_{0}}\left(\boldsymbol{x}_{i}, \boldsymbol{y}_{i}\right)=\frac{1}{\rho^{5 / 2}} \sum_{\gamma_{i}} F_{\gamma_{i}}^{n_{0} J_{0}}(\rho) \mathcal{Y}_{\gamma_{i}}^{J_{0} M_{0}}\left(\Omega_{i}\right)
$$

where

$$
\mathcal{Y}_{\gamma_{i}}^{J_{0} M_{0}}\left(\Omega_{i}\right)=\phi_{K}^{\left(\ell_{x_{i}}, \ell_{y_{i}}\right)}\left(\alpha_{i}\right)\left[\left|\ell_{x_{i}}, s_{x_{i}}, j_{x_{i}}\right\rangle \otimes\left|\ell_{y_{i}}, s_{i}, j_{y_{i}}\right\rangle\right]^{J_{0} M_{0}} \text {, }
$$

with $\gamma_{i} \equiv\left\{K, \ell_{x_{i}}, s_{x_{i}}, j_{x_{i}}, \ell_{y_{i}}, j_{y_{i}}\right\}$ and with $\phi_{K}^{\left(\ell_{x_{i}}, \ell_{y_{i}}\right)}\left(\alpha_{i}\right)$ being the usual function of the hyperangle $\alpha_{i}$ entering in the definition of the hyperspherical harmonics [13]. The functions (24) reduce to the usual hyperspherical harmonics for particles without spin.

With the definition (21), the two-body potential operator $\hat{V}_{2 b}^{(i)}$ acting on a term of the basis $\mathcal{Y}_{\gamma_{i}}^{J_{0} M_{0}}\left(\Omega_{i}\right)$ (written in the Jacobi set $i$ ) leads to

$$
\hat{V}_{2 b}^{(i)} \mathcal{Y}_{\gamma_{i}}^{J_{0} M_{0}}\left(\Omega_{i}\right)=V_{i}^{\left(\ell_{\chi_{i}}, s_{x_{i}}, x_{x_{i}}\right)}\left(x_{i}\right) \hat{P}_{i} \mathcal{Y}_{\gamma_{i}}^{J_{0} M_{0}}\left(\Omega_{i}\right) .
$$

Equation (23) permits us to write Eq. (22) as

$$
\begin{aligned}
S_{1}^{(2 b, i)}= & \sum_{\mu} \int d \rho \sum_{\gamma_{i}} \sum_{\gamma_{i}^{\prime}} F_{\gamma_{i}}^{n_{0} J_{0}}(\rho) F_{\gamma_{i}^{\prime}}^{n_{0} J_{0}}(\rho) \\
& \times\left[\left\langle\mathcal{Y}_{\gamma_{i}}^{J_{0} M_{0}}\left|O_{\mu}^{\lambda \dagger} \hat{V}_{2 b}^{(i)} O_{\mu}^{\lambda}\right| \mathcal{Y}_{\gamma_{i}^{\prime}}^{J_{0} M_{0}}\right\rangle\right. \\
& \left.-\left\langle\mathcal{Y}_{\gamma_{i}}^{J_{0} M_{0}}\left|O_{\mu}^{\lambda \dagger} O_{\mu}^{\lambda} \hat{V}_{2 b}^{(i)}\right| \mathcal{Y}_{\gamma_{i}^{\prime}}^{J_{0} M_{0}}\right\rangle\right] .
\end{aligned}
$$

Inserting in the two matrix elements in Eq. (26) the unity operator

$$
\mathbf{1}=\sum_{\gamma_{i}^{\prime \prime}} \sum_{J^{\prime \prime} M^{\prime \prime}}\left|\mathcal{Y}_{\gamma_{i}^{\prime \prime}}^{J^{\prime \prime} M^{\prime \prime}}\left(\Omega_{i}\right)\right\rangle\left\langle\mathcal{Y}_{\gamma_{i}^{\prime \prime}}^{J^{\prime \prime} M^{\prime \prime}}\left(\Omega_{i}\right)\right|
$$

in between $O_{\mu}^{\lambda \dagger}$ and $\hat{V}_{2 b}^{(i)}$, and $O_{\mu}^{\lambda \dagger}$ and $O_{\mu}^{\lambda}$, respectively, and making use of Eq. (25), we immediately get the final expression:

$$
\begin{aligned}
S_{1}^{(2 b, i)}= & \sum_{\mu} \int \mathrm{d} \rho \sum_{\gamma_{i}} \sum_{\gamma_{i}^{\prime}} F_{\gamma_{i}}^{n_{0} J_{0}}(\rho) F_{\gamma_{i}^{\prime}}^{n_{0} J_{0}}(\rho) \\
& \times \sum_{\gamma_{i}^{\prime \prime}} \sum_{J^{\prime \prime} M^{\prime \prime}}\left\langle\mathcal{Y}_{\gamma_{i}}^{J_{0} M_{0}}\left(\Omega_{i}\right)\left|O_{\mu}^{\lambda \dagger}\right| \mathcal{Y}_{\gamma_{i}^{\prime \prime}}^{J^{\prime \prime} M^{\prime \prime}}\left(\Omega_{i}\right)\right\rangle \\
& \times\left\langle\mathcal{Y}_{\gamma_{i}^{\prime \prime}}^{J^{\prime \prime} M^{\prime \prime}}\left(\Omega_{i}\right)\right|\left(V_{i}^{\left(\ell_{x_{i}}^{\prime \prime}, s_{x_{i}}^{\prime \prime}, j_{x_{i}}^{\prime \prime}\right)}\left(x_{i}\right) \hat{P}_{i} O_{\mu}^{\lambda}\right. \\
& \left.-V_{i}^{\left(\ell_{x_{i}}^{\prime}, s_{x_{i}}^{\prime}, j_{x_{i}}^{\prime}\right)}\left(x_{i}\right) O_{\mu}^{\lambda} \hat{P}_{i}\right)\left|\mathcal{Y}_{\gamma_{i}^{\prime}}^{J_{0} M_{0}}\left(\Omega_{i}\right)\right\rangle .
\end{aligned}
$$


When the $V_{i}$ functions are independent of the partial wave, this equation can be written in a more compact way as

$$
\begin{aligned}
S_{1}^{(2 b, i)}= & \frac{1}{2} \sum_{\mu} \int d \rho \sum_{\gamma_{i}} \sum_{\gamma_{i}^{\prime}} F_{\gamma_{i}}^{n_{0} J_{0}}(\rho) F_{\gamma_{i}^{\prime}}^{n_{0} J_{0}}(\rho) \\
& \times\left\langle\mathcal{Y}_{\gamma_{i}}^{J_{0} M_{0}}\left|V_{i}\left(x_{i}\right)\left[\left[O_{\mu}^{\lambda \dagger}, \hat{P}_{i}\right], O_{\mu}^{\lambda}\right]\right| \mathcal{Y}_{\gamma_{i}^{\prime}}^{J_{0} M_{0}}\right\rangle,
\end{aligned}
$$

which is trivially zero for the central part of the two-body potential $\left(\hat{P}_{i}=1\right)$ and for the spin-spin term (since $O_{\mu}^{\lambda}$ does not depend on the spin and therefore commutes with the spin-spin operator). The same result is obtained for the tensor operator, which depends only on coordinates and spin operators. For the spin-orbit term $\left(\ell_{x} \cdot s_{x}=\ell_{+} s_{-}+\ell_{-} s_{+}+\ell_{z} s_{z}\right)$ one has the same result, since $\ell_{+}, \ell_{-}$, or $\ell_{z}$ applied on $O_{\mu}^{\lambda}$ is proportional to $O_{\mu+1}^{\lambda}, O_{\mu-1}^{\lambda}$, or $O_{\mu}^{\lambda}$, and therefore each of the three terms in $\boldsymbol{\ell}_{x} \cdot \boldsymbol{s}_{x}$ double commutes with $O_{\mu}^{\lambda}$.

Thus, for two-body interactions independent of the partial waves and containing the usual spin operators one has $S_{1}^{(2 b)}=0$.

If the two-body potentials are partial-wave dependent, but contain only central, spin-spin $\left(\hat{P}_{i}=\boldsymbol{s}_{j} \cdot \boldsymbol{s}_{k}\right)$ and spin-orbit $\left(\hat{P}_{i}=\boldsymbol{\ell}_{x_{i}} \cdot \boldsymbol{s}_{x_{i}}\right)$ terms, because these operators are diagonal in the basis $\left\{\left|\ell_{x_{i}}, s_{x_{i}}, j_{x_{i}}, m_{x_{i}}\right\rangle\right\}$, one then has

$$
\hat{P}_{i} \mathcal{Y}_{\gamma_{i}}^{J_{0} M_{0}}\left(\Omega_{i}\right)=f_{\ell_{x_{i}}, s_{x_{i}}}^{j_{x_{i}}} \mathcal{Y}_{\gamma_{i}}^{J_{0} M_{0}}\left(\Omega_{i}\right),
$$

where $f_{\ell_{x_{i}}, s_{x_{i}}}^{j_{x_{i}}}=1$ for the central part of the potential, $f_{\ell_{x_{i}}, s_{x_{i}}}^{j_{x_{i}}}=\left[s_{x_{i}}\left(s_{x_{i}}+1\right)-s_{j}\left(s_{j}+1\right)-s_{k}\left(s_{k}+1\right)\right] / 2$ for the spin-spin part, and $f_{\ell_{x_{i}}, s_{x_{i}}}^{j_{x_{i}}}=\left[j_{x_{i}}\left(j_{x_{i}}+1\right)-\ell_{x_{i}}\left(\ell_{x_{i}}+1\right)-\right.$ $\left.s_{x_{i}}\left(s_{x_{i}}+1\right)\right] / 2$ for the spin-orbit part. Equation (28) can then be written for this particular case as

$$
\begin{aligned}
S_{1}^{(2 b, i)}= & \sum_{\mu} \int \mathrm{d} \rho \sum_{\gamma_{i}} \sum_{\gamma_{i}^{\prime}} F_{\gamma_{i}}^{n_{0} J_{0}}(\rho) F_{\gamma_{i}^{\prime}}^{n_{0} J_{0}}(\rho) \\
& \times \sum_{\gamma_{i}^{\prime \prime}} \sum_{J^{\prime \prime} M^{\prime \prime}}\left\langle\mathcal{Y}_{\gamma_{i}}^{J_{0} M_{0}}\left(\Omega_{i}\right)\left|O_{\mu}^{\lambda \dagger}\right| \mathcal{Y}_{\gamma_{i}^{\prime \prime}}^{J^{\prime \prime} M^{\prime \prime}}\left(\Omega_{i}\right)\right\rangle \\
& \times\left\langle\mathcal{Y}_{\gamma_{i}^{\prime \prime}}^{J^{\prime \prime} M^{\prime \prime}}\left(\Omega_{i}\right)\right|\left(V_{i}^{\left(\ell_{x_{i}}^{\prime \prime}, s_{x_{i}}^{\prime \prime}, j_{x_{i}}^{\prime \prime}\right)}\left(x_{i}\right) f_{\ell_{x_{i}}^{\prime \prime}, s_{x_{i}}^{\prime \prime}}^{j_{x_{i}}^{\prime \prime}}\right. \\
& \left.-V_{i}^{\left(\ell_{x_{i}}^{\prime}, s_{x_{i}}^{\prime}, j_{x_{i}}^{\prime}\right)}\left(x_{i}\right) f_{\ell_{x_{i}}^{\prime}, s_{x_{i}}^{\prime}}^{j_{x_{i}}^{\prime}}\right) O_{\mu}^{\lambda}\left|\mathcal{Y}_{\gamma_{i}^{\prime}}^{J_{0} M_{0}}\left(\Omega_{i}\right)\right\rangle .
\end{aligned}
$$

It is important to keep in mind that the operator $O_{\mu}^{\lambda}$ has three terms [see Eq. (6)], each expressed in one of the three sets of Jacobi coordinates. When inserted in Eq. (31), the two terms in $O_{\mu}^{\lambda}$ differing from the set of Jacobi coordinates $i$ must be transformed into this set by use of Eq. (5).

When only one of the three particles is charged the operator $O_{\mu}^{\lambda}$ reduces to one term. A partial-wave dependence in the interaction between the charged particle and any of the other two will produce a nonvanishing contribution to the energy-weighted sum rule according to Eq. (31). However, if the only partial-wave dependence appears in the two-body potential between the two neutral particles then $S_{1}^{(2 b)}=0$. This is because the $O_{\mu}^{\lambda}$ operator then automatically is written in the same Jacobi set as the angular functions in Eq. (31). The operator is then independent of $\Omega_{x_{i}}$, and the integral over these angles in the last matrix element of Eq. (31) vanishes unless $\ell_{x_{i}}^{\prime \prime}=\ell_{x_{i}}^{\prime}, s_{x_{i}}^{\prime \prime}=s_{x_{i}}^{\prime}$, and $j_{x_{i}}^{\prime \prime}=j_{x_{i}}^{\prime}$ and therefore the full matrix element vanishes.

The integrals over $\Omega_{x_{i}}$ and $\Omega_{y_{i}}$ in the two matrix elements that appear in Eq. (31) can be calculated analytically, because $x_{i}=\rho \sin \alpha_{i}$, and therefore the two-body potentials are independent of the angles $\Omega_{x_{i}}$ and $\Omega_{y_{i}}$. The expressions for these two matrix elements are given as Eq. (A6) of the Appendix for the particular case of particles without spin.

As an example we consider dipole excitations $(\lambda=1)$ in a system of three spin-zero particles with equal mass $m=4 m_{N}$, where $m_{N}$ is the nucleon mass, and where two particles are neutral and one particle has a charge equal to twice the proton charge. We consider only $s$ and $p$ waves in the calculation. The two-body interactions are taken to be Gaussians $\left[V_{s, p}(r)=W_{s, p} e^{-r^{2} / b^{2}}\right]$ with equal range $b$ for $s$ and $p$ waves. We have constructed a $0^{+}$ground state with a very large contribution of $s$ waves and only a few percent $p$ waves. This has been done by taking $b=2.98 \mathrm{fm}, W_{s}=W_{p}=$ $-0.18 \mathrm{MeV}$ for the interaction between the two neutral particles, and $W_{s}=W_{p}=-1.18 \mathrm{MeV}$ for the interaction between the charged particle and one of the neutral ones. The binding energy of the $0^{+}$state is $-10.4 \mathrm{MeV}$. According to Eq. (31), since only terms with $\ell_{x_{i}}^{\prime \prime} \neq \ell_{x_{i}}^{\prime}$ contribute $\left(s_{x_{i}}^{\prime \prime}=\right.$ $s_{x_{i}}^{\prime}=0, j_{x_{i}}^{\prime \prime}=\ell_{x_{i}}^{\prime \prime}, j_{x_{i}}^{\prime}=\ell_{x_{i}}^{\prime}$, and $\left.f_{\ell_{x_{i}}, s_{x_{i}}}^{j_{x_{i}}}=1\right)$, the contribution $S_{1}^{(2 b)}$ to the energy-weighted sum rule is proportional to $\Delta W=W_{s}-W_{p}$, where $W_{s}$ and $W_{p}$ refer to the strengths of the interactions between the charged and the neutral particle.

In Fig. 1, the dashed line ( + signs) shows $S_{1}^{(2 b)}$ as a function of $\Delta W$ when the strength of the $s$-wave potential between the charged and neutral particles $\left(W_{s}\right)$ is kept fixed and $W_{p}$ is changed. Since the $p$-wave contribution to the ground-state wave function is insignificant, a small variation in the strength of the $p$-wave potential only slightly modifies the ground-state radial wave functions $F_{\gamma}^{n_{0} J_{0}}(\rho)$. Therefore, the behavior of $S_{1}^{(2 b)}$ is almost perfectly linear with $\Delta W$. However, if we modify $\Delta W$ by keeping $W_{p}$ fixed while changing $W_{s}$, the radial wave functions are much more sensitive to a change in the $s$-wave two-body potential, since the $s$-waves dominate. Therefore $S_{1}^{(2 b)}$ is not a completely linear function of $\Delta W$ as seen by the solid line ( $\times$ signs in the figure). When $\Delta W=0$, the total value of $S_{1}$ for this particular case is $S_{1}=9.90 e^{2} \mathrm{MeV} \mathrm{fm}^{2}$, which means that the contribution from $\Delta W \neq 0$ can be of comparable size (see Fig. 1).

\section{C. $J^{\pi}$-Dependent Three-Body Potentials}

When performing three-body calculations it is quite usual to employ effective three-body forces to fine-tune the energies of the computed states. Very often different three-body forces are used to place the lowest state with given angular momentum and parity $J^{\pi}$ at the correct energy. This means that these three-body potentials usually depend on $J^{\pi}$. In this section we investigate the additional contribution $S_{1}^{(3 b)}$ to the energyweighted sum rule arising from this kind of three-body potentials. With these assumptions the three-body potential 


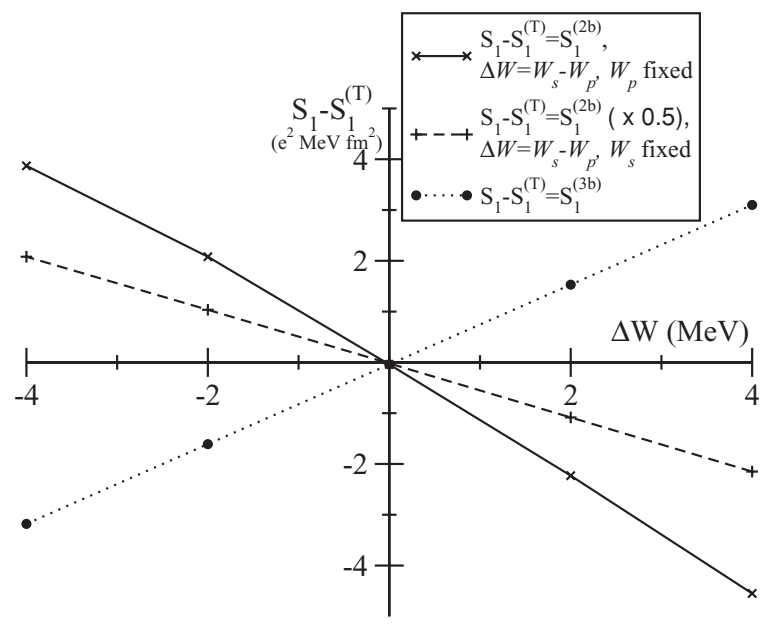

FIG. 1. For dipole excitations in a system of three particles with equal mass ( $m=4$ times the nucleon mass), and only one of them with a charge (equal to twice the proton charge), the figure shows the variation of $\left(S_{1}-S_{1}^{(T)}\right)$ as a function of the strength difference $(\Delta W)$ between the Gaussian $s$ - and $p$-wave two-body potentials of equal range (see text). The solid and dashed lines give the results when only the $s$-wave strength and only the $p$-wave strength are changed, respectively (the dashed line has been multiplied by a factor 0.5 to be more easily distinguished from the solid line). The dotted line shows the same variation as a function of the strength difference between the Gaussian three-body forces in the excited $\left(1^{-}\right)$and ground $\left(0^{+}\right)$ states when the three-body potentials have equal range, when only the strength of the $1^{-}$states is changed, and when the two-body potentials are $\ell$-independent.

operator can be written as

$$
\hat{V}_{3 b}=\sum_{J \pi M} \sum_{n_{\left(J^{\pi}\right)}} V_{3 b}^{\left(J^{\pi}\right)}(\rho)\left|n_{\left(J^{\pi}\right)} J^{\pi} M\right\rangle\left\langle n_{\left(J^{\pi}\right)} J^{\pi} M\right|,
$$

where $n_{\left(J^{\pi}\right)}$ refers to all the additional quantum numbers needed to specify each of the three-body states with total angular momentum and parity $J^{\pi}$. Following Eq. (14) we can write

$$
\begin{aligned}
S_{1}^{(3 b)}= & \sum_{\mu}\left[\left\langle n_{0} J_{0}^{\pi_{0}} M_{0}\left|O_{\mu}^{\lambda \dagger} \hat{V}_{3 b} O_{\mu}^{\lambda}\right| n_{0} J_{0}^{\pi_{0}} M_{0}\right\rangle\right. \\
& \left.-\left\langle n_{0} J_{0}^{\pi_{0}} M_{0}\left|O_{\mu}^{\lambda \dagger} O_{\mu}^{\lambda} \hat{V}_{3 b}\right| n_{0} J_{0}^{\pi_{0}} M_{0}\right\rangle\right],
\end{aligned}
$$

where we explicitly labeled the initial state by its parity $\pi_{0}$.

Substituting now Eq. (32) into Eq. (33) and inserting the unity operator between $O_{\mu}^{\lambda \dagger}$ and $O_{\mu}^{\lambda}$ in the last matrix element, we finally get

$$
\begin{aligned}
S_{1}^{(3 b)}= & \sum_{\mu} \sum_{J \pi M} \sum_{n_{\left(J^{\pi}\right)}}\left\langle n_{\left(J^{\pi}\right)} J^{\pi} M\left|O_{\mu}^{\lambda}\right| n_{0} J_{0}^{\pi_{0}} M_{0}\right\rangle\left\langle n_{0} J_{0}^{\pi_{0}} M_{0}\right| \\
& \times O_{\mu}^{\lambda \dagger}\left(V_{3 b}^{\left(J^{\pi}\right)}-V_{3 b}^{\left(J_{0}^{\pi_{0}}\right)}\right)\left|n_{\left(J^{\pi}\right)} J^{\pi} M\right\rangle
\end{aligned}
$$

which gives the contribution to the energy-weighted sum rule from $J^{\pi}$-dependent three-body potentials. This contribution vanishes when the three-body interactions are $J^{\pi}$-independent.
For the special case in which the ground state has $J_{0}=0$ the expression simplifies to

$$
S_{1}^{(3 b)}=\sum_{\mu}\left\langle\left. n_{0} 0^{\pi_{0}} 0|| O_{\mu}^{\lambda}\right|^{2}\left(V_{3 b}^{\left(\lambda^{\pi}\right)}(\rho)-V_{3 b}^{\left(0^{\pi_{0}}\right)}(\rho)\right) \mid n_{0} 0^{\pi_{0}} 0\right\rangle,
$$

where $\pi=\pi_{0}(-1)^{\lambda}$, which, except for the difference between the three-body potentials, is similar to Eq. (9). Therefore, the analytic expression of $S_{1}^{(3 b)}$ for $J_{0}=0$ is given by Eq. (12), but with an additional factor equal to the difference between the three-body potentials inserted in the last matrix element.

In Fig. 1 the dotted line (with circles) shows $S_{1}^{(3 b)}$ for the same system and the same transition as for the $S_{1}^{(2 b)}$ case. We have taken the $\ell$-independent two-body potentials used in Sec. IV B as a starting point, meaning that $S_{1}^{(3 b)}=S_{1}-S_{1}^{(T)}$. The result is shown as a function of the strength difference $(\Delta W)$ between the Gaussian effective three-body forces used to compute the $1^{-}$excited states and the $0^{+}$ground state. The range of the three-body force is the same $(6.0 \mathrm{fm})$ for $0^{+}$and $1^{-}$. The variation in $\Delta W$ is obtained by changing the strength in the three-body force for the $1^{-}$excited states. Then the ground-state wave function remains unchanged. As a consequence, according to Eq. (34), and as demonstrated by the dotted line in the figure, $S_{1}^{(3 b)}$ depends linearly on $\Delta W$. The contribution from $S_{1}^{(3 b)}$ can be of comparable size to the value, $S_{1}=9.90 e^{2} \mathrm{MeV} \mathrm{fm}^{2}$, for angular-momentum-independent potentials.

\section{A REALISTIC CASE: DIPOLE EXCITATIONS IN ${ }^{6} \mathrm{He}$}

The main properties of the Borromean two-neutron halo nuclei are well reproduced by describing them as three-body systems made by an inert core surrounded by two neutrons. The characteristic feature of these nuclei is their large spatial extension, which is responsible for the large values of the breakup cross sections after electromagnetic excitation. This can be easily envisaged from Eqs. (13) and (20), which depend directly on the size of the system. For this reason, electromagnetic excitations of two-neutron halo nuclei have attracted a lot of attention, especially dipole excitations, which is the dominating multipolarity for such excitations.

In this section we investigate dipole excitations in ${ }^{6} \mathrm{He}$ $(\alpha+n+n)$, which is one of the most prominent examples of Borromean two-neutron halo nuclei. We compute the threebody states by use of the hyperspheric adiabatic expansion method [13]. The neutron-neutron and $\alpha$-neutron interactions are the ones used for instance in Ref. [17]. The computed bound ground state $\left(0^{+}\right)$has a two-neutron separation energy matching the experimental value of $-0.97 \pm 0.04 \mathrm{MeV}$. This is achieved with a Gaussian effective three-body force with range $2.9 \mathrm{fm}$ and strength $-7.55 \mathrm{MeV}$. The continuum $1^{-}$ states have been discretized by use of a box boundary condition at $\rho_{\max }=50 \mathrm{fm}$. 


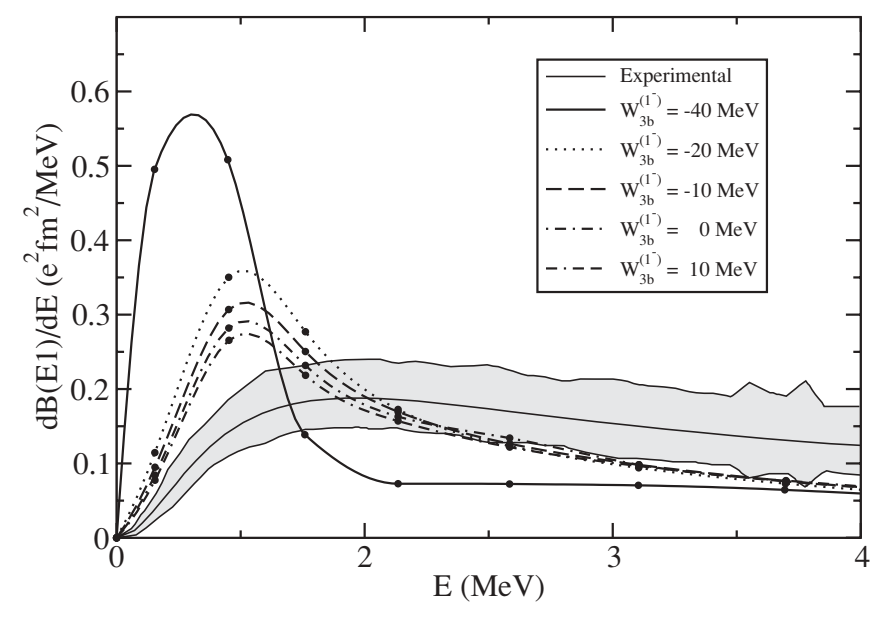

FIG. 2. Computed (circles) dipole strength function for ${ }^{6} \mathrm{He}$. The curve through the computed points is obtained by cubic interpolation. Gaussian three-body forces are used to obtain the ground $\left(0^{+}\right)$and excited $\left(1^{-}\right)$three-body states. The range of the Gaussian $(2.9 \mathrm{fm})$ is the same for both angular momenta. The different curves show the results obtained with different values of the strength $\left(W_{3 b}^{\left(1^{-}\right)}\right)$of the three-body force for the $1^{-}$states. The experimental data (shaded area) are from Ref. [18].

\section{A. Dipole Strength Function}

The transition probability $\mathcal{B}$ from the ground state to one of the box discretized continuum states is given in Eq. (7). To obtain a smooth distribution from the discretized continuum we use the finite-energy interval approximation to the strength function,

$$
\frac{d \mathcal{B}}{d E} \approx \frac{\Delta \mathcal{B}}{\Delta E},
$$

where $\Delta E$ is the size of the given energy interval, and $\Delta \mathcal{B}$ is the sum of the transition probabilities into the states whose energies fall into this interval. These values are then plotted as function of the central energy values of the intervals.

The interval should be of a reasonable size (i.e., large enough to provide a smooth function but small enough not to wash out the desired structure). In practice we have used bins with centers at $0.3,0.9,1.5,2.3,3.2,4.2,5.4,6.7$, and 8.2 $\mathrm{MeV}$ and a standard cubic interpolation to smooth the curve.

In Fig. 2 we compare the computed smoothed dipole strength function for ${ }^{6} \mathrm{He}$ for different Gaussian three-body potentials in the excited $1^{-}$states with the measured distribution [18]. A strongly attractive three-body potential produces a pronounced low-lying peak, which for even stronger attraction would turn into a bound state. Thus the three-body potential should at least be less attractive than that corresponding to $W_{3 b}^{\left(1^{-}\right)}=-40 \mathrm{MeV}(b=2.9 \mathrm{fm})$. For moderately attractive three-body potentials we observe an increase from zero at threshold to a peak value at around $1 \mathrm{MeV}$ followed by a relatively fast decrease toward zero at higher energies. This is consistent with the calculations in Refs. [8] and [19]. Compared to the experiment [18], the theory overestimates the strength at around $1 \mathrm{MeV}$ and, consistent with the sum rule, underestimates the strength at higher energies. Apparently
TABLE II. Non-energy-weighted $\left(S_{0}\right)$ and energy-weighted $\left(S_{1}\right)$ dipole sum rule values for ${ }^{6} \mathrm{He}$. The fourth column gives the contribution to $S_{1}$ from the kinetic energy operator $\left(S_{1}^{(T)}\right)$. The last column $\left(S_{1} / S_{0}\right)$ is an average dipole resonance energy. Shown are the experimental and computed sum rule results for states below energies of 5 and $10 \mathrm{MeV}$, respectively. The last row gives the converged results including all excitations. The experimental data are from Ref. [18]. The $S_{0}$ values are given in units of $e^{2} \mathrm{fm}^{2}$ and $S_{1}$ and $S_{1}^{(T)}$ are in units of $e^{2} \mathrm{fm}^{2} \mathrm{MeV}$. The average dipole resonance energy $S_{1} / S_{0}$ is given in $\mathrm{MeV}$.

\begin{tabular}{lcccc}
\hline \hline & $S_{0}$ & $S_{1}$ & $S_{1}^{(T)}$ & $S_{1} / S_{0}$ \\
\hline$E^{*} \leqslant 5 \mathrm{MeV}$ (expt.) & $0.59 \pm 0.12$ & $1.9 \pm 0.4$ & - & $3.22 \pm 0.94$ \\
$E^{*} \leqslant 5 \mathrm{MeV}$ (theor.) & 0.66 & 1.94 & - & 2.94 \\
$E^{*} \leqslant 10 \mathrm{MeV}$ (expt.) & $1.2 \pm 0.2$ & $6.4 \pm 1.3$ & - & $5.3 \pm 1.4$ \\
$E^{*} \leqslant 10 \mathrm{MeV}$ (theor.) & 1.01 & 4.43 & - & 4.39 \\
Converged & 1.25 & 8.26 & 4.95 & 6.61 \\
\hline \hline
\end{tabular}

a significant three-body repulsion in the $1^{-}$channel would approach the experimental data presented with relatively large error bars.

\section{B. Sum Rule Results}

The second and third columns of Table II give the nonenergy-weighted $\left(S_{0}\right)$ and energy-weighted $\left(S_{1}\right)$ dipole sum rule strengths. The experimental data, available from Ref. [18], are given in the first and third rows, including states of energies below 5 and $10 \mathrm{MeV}$, respectively. The corresponding theoretical values are obtained numerically directly from the first row of Eq. (9) and Eq. (15), and they are given in the second and fourth rows of the table.

We can see that the computed results for $S_{0}$ agree very well with the experimental values when the sum over the excited states in Eq. (9) is restricted to energies below 5 and $10 \mathrm{MeV}$, respectively. As even higher energies are included the value of $S_{0}$ converges to the result given in the last row of the table, which agrees with the expected result obtained from Eq. (13). The converged value is already reached with an energy limit of about $40 \mathrm{MeV}$.

Essentially the same sort of agreement happens for $S_{1}$. The computed values agree reasonably well with the experimental ones. Also the result obtained for energies below $10 \mathrm{MeV}$ is still clearly below the converged value, which requires integration up to energies at least of about $60 \mathrm{MeV}$. The converged value for $S_{1}$ clearly disagrees with the result provided by Eq. (20), where only the kinetic energy contribution is considered. This value is given in the fourth column of Table II. It is important to note that the computed results given in the table have been obtained by using the same effective threebody force for the $0^{+}$ground state and the $1^{-}$excited states. This means that the difference between the converged $S_{1}$ value and $S_{1}^{(T)}$ is exclusively due to the effect of the $\ell$-dependence of the two-body $\alpha$-neutron potentials [see Eq. (31)]. As seen in the table, this effect is far from being negligible.

The last column in Table II shows the ratio between the energy-weighted and non-energy-weighted sum rules, which is interpreted as an average energy of the soft dipole mode. 


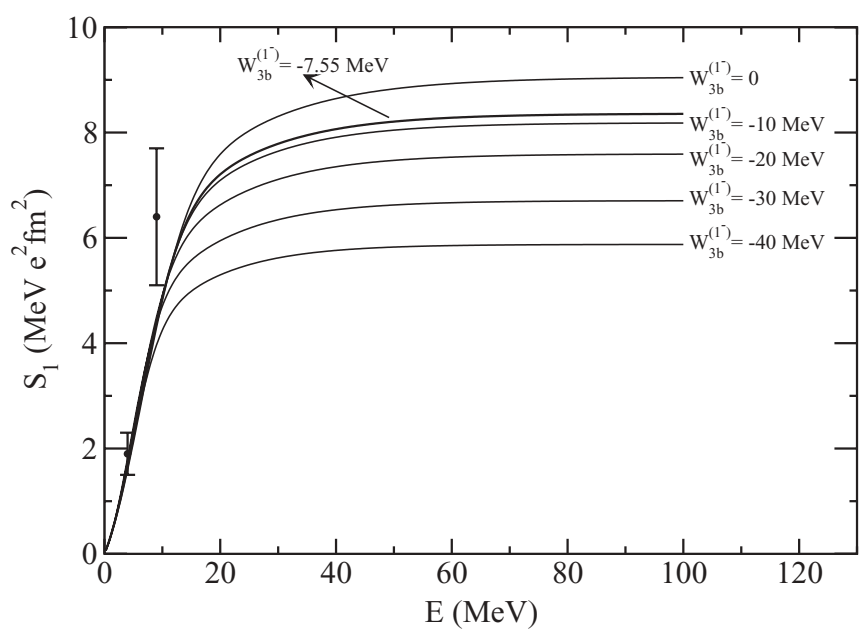

FIG. 3. Computed dipole sum rule strength $S_{1}$ for ${ }^{6} \mathrm{He}$ as a function of the maximum energy allowed above threshold. Gaussian three-body forces are used to obtain the ground $\left(0^{+}\right)$and excited $\left(1^{-}\right)$ three-body states. The range of the Gaussian is the same $(2.9 \mathrm{fm})$ for all the states. The different curves show the results obtained with different values of the strength $\left(W_{3 b}^{\left(1^{-}\right)}\right)$of the three-body force for the $1^{-}$states. The thick curve is the result with the same three-body force for the ground and excited states. The experimental data are from Ref. [18].

The value of $6.6 \mathrm{MeV}$ obtained after reaching convergence in $S_{0}$ and $S_{1}$ is consistent with previous results, such as those in Refs. [20,21], where a value of about $5 \mathrm{MeV}$ also is obtained. In Ref. [19] a clearly smaller value is given (3.8 MeV), very likely because these authors used $S_{1}^{(T)}$ instead of the full $S_{1}$ in the computation of the ratio. In any case these rather large variations illustrate how important it is in practice to use the correct sum rules in such estimates.

So far all the calculations of the $S_{1}$ strength have been performed with the same effective three-body force for the ground state and the $1^{-}$excited states. This force was adjusted to fit the experimental two-neutron separation energy in the $0^{+}$-state. However, for the $1^{-}$states the interaction might be different and an additional contribution to the $S_{1}$ strength would appear as seen in Eq. (35).

In Fig. 3 we show the dipole sum rule strength $S_{1}$ for ${ }^{6} \mathrm{He}$ as a function of the maximum energy allowed above threshold. We have considered Gaussian three-body forces with a range of $2.9 \mathrm{fm}$. For the ground state $\left(0^{+}\right)$a strength of $-7.55 \mathrm{MeV}$ has been used. When the excited states are obtained with the same three-body force, $S_{1}$ behaves as shown by the thick solid line in the figure. This calculation corresponds to the numbers quoted in Table II. When the strength of the three-body force used for the $1^{-}$states is changed, $S_{1}^{(3 b)}$ depends linearly on the strength difference.

The thin solid lines in the figure show $S_{1}$ for different values of the strength $\left(W_{3 b}^{\left(1^{-}\right)}\right)$of the three-body force for the $1^{-}$states. This strength has been changed from 0 to $-40 \mathrm{MeV}$, which is at the limit of producing a low-lying narrow $1^{-}$resonance in ${ }^{6} \mathrm{He}$ (understood as a pole of the $S$ matrix). As seen in the figure, the deeper the three-body potential, the smaller is the value of $S_{1}$. The converged value can change significantly with the three-body force. The result obtained with a strength of $-40 \mathrm{MeV}$ is about $33 \%$ smaller than that obtained for $W_{3 b}^{\left(1^{-}\right)}=$ 0 . Therefore, the value of the average dipole resonance energy (the ratio between the values of the two sum rules) also changes substantially with the three-body force, ranging between 7.2 $\mathrm{MeV}$ when $W_{3 b}^{\left(1^{-}\right)}=0$ and $4.7 \mathrm{MeV}$ when $W_{3 b}^{\left(1^{-}\right)}=$ $-40 \mathrm{MeV}$.

As seen in the figure, all the curves agree well with the experimental value obtained for states below a maximum energy of $5 \mathrm{MeV}$. When this maximum energy is $10 \mathrm{MeV}$, all the computed curves are below the experimental value. Although this experimental value has a rather large error bar, it is clear from the figure that the smaller strengths in the three-body force for the $1^{-}$states are closer to the experimental value. This tendency is consistent with the fact that a $1^{-}$resonance has not been found experimentally since a strength weaker than about $-30 \mathrm{MeV}$ also excludes such a state in computations. Extension to include higher energies in the experiment would allow distinction between the values obtained for different strengths. Probably ${ }^{6} \mathrm{He}$ is a very favorable system for this investigation because the core excitations are expected to be negligible.

\section{SUMMARY AND CONCLUSIONS}

We have derived general expressions for the non-energyweighted and energy-weighted cluster sum rules for excitations of three-body systems. We consider transitions arising from electric multipole operators of any order and for which each of the constituent particles (clusters) may have a finite charge. The most obvious nuclear applications are in systems close to three-body thresholds where three-body clusterization frequently seems to be a dominating part of the structure. This is also the region where the spatially extended and weakly bound halos appear.

Accurate calculations of three-body wave functions require in general decomposition into Faddeev components either by directly solving the Faddeev equations or by a variational procedure including similar components expressed in the different Jacobi coordinates. Derivation of the non-energyweighted sum rule only relies on the use of a complete set of intermediate wave functions. Therefore only matrix elements of the multipole operators enter into the expressions whereas the interactions disappear altogether, except of course indirectly through the properties of the excited continuum states. However, the properties of the wave functions are essential, and in particular the different Faddeev components give rise to crucial interference effects when more than one particle carries a charge.

Such interference effects are also crucial for the energyweighted sum rule where in addition also the properties of the interactions are essential. This sum rule is traditionally derived as a double commutator between the Hamiltonian and two multipole operators. Usually then only the second-order derivatives from the kinetic energy operator contribute whereas the potentials including the spin-orbit terms commute with the multipole operators and lead to vanishing contributions. However, when the interactions are angular momentum dependent, the double commutator does not vanish because the 
multipole operators themselves also carry angular momentum. These contributions must therefore be computed and included in the sum rule estimates. Still the character of the sum rule remains in the sense that, no matter how the excitations are distributed, they must add up to the value given by the sum rule, which only depends on properties of the ground state and the interactions.

The angular momentum dependence and the subsequent contributions to the sum rule are separated into terms arising from the two- and three-body potentials, which often in accurate three-body computations depend on angular momentum. A possible sequence to determine appropriate potentials could be as follows: First, adjust the two-body potentials independently for each partial wave to known two-body bound or continuum properties. Second, fine-tune the three-body state computed with the two-body potentials to a desired energy by adding a short-range three-body potential with as little structure as possible to maintain the properties provided by the two-body interactions. Both types of angular momentum dependence are important as they turn out to give substantial contributions to the ordinary kinetic energy contribution to the sum rule.

To assess numerically the relative importance of these new sum rule contributions we investigate the electric dipole excitations of the ground state of the well-known halo nucleus ${ }^{6} \mathrm{He}$. We first notice that the strength distribution has a peak at around $1 \mathrm{MeV}$, falls off at higher energies, and in practice reaches zero at about $60 \mathrm{MeV}$. The contribution to the energy-weighted sum rule from the two-body potentials amounts to two-thirds of the kinetic energy contribution.

The contribution from the three-body potential depends on an expectation value of the difference between those potentials for ground- and excited-state angular momenta of 0 and 1 , respectively. Thus for state-independent but finite three-body potentials we arrive at the established result of zero contribution. However, the sensitivity to the difference in these three-body potentials is significant. Realistic potentials give estimates of up to $30 \%$ of the kinetic energy value. This is then also an estimate of the sensitivity of the soft dipole mode to the three-body potential.

In conclusion, we have generalized the energy-weighted and non-energy-weighted cluster sum rules for electric multipole transitions to angular momentum two- and three-body interactions. The additional contributions can be comparable in size to the ordinary terms arising from the kinetic energy operator.

\section{ACKNOWLEDGMENTS}

This work was partly supported by funds provided by DGI of MEC (Spain) under contract no. FIS2005-00640. One of us (R.D.) acknowledges support by a predoctoral I3P fellowship from CSIC and the European Social Fund.

\section{APPENDIX A: INTERMEDIATE EXPRESSIONS AND FORMULAS}

In this appendix we give some of the intermediate expressions obtained when deriving Eq. (19).
The commutator between the electric multipole operator $O_{\mu}^{\lambda \dagger}$ and the kinetic energy operator $T$ can be written as

$$
\begin{aligned}
{\left[O_{\mu}^{\lambda \dagger}, T\right] } & =-\sum_{k=1}^{3} z_{k} \frac{\hbar^{2}}{2 m_{N}}\left[\left|\boldsymbol{r}_{k}-\boldsymbol{R}\right|^{\lambda} Y_{\lambda, \mu}^{*}\left(\Omega_{y_{k}}\right), \Delta_{y_{k}}\right] \\
& =\sum_{k=1}^{3} z_{k} \frac{\hbar^{2}}{m_{N}} \nabla_{y_{k}}\left(\left|\boldsymbol{r}_{k}-\boldsymbol{R}\right|^{\lambda} Y_{\lambda, \mu}^{*}\left(\Omega_{y_{k}}\right)\right) \cdot \nabla_{y_{k}},
\end{aligned}
$$

where we have used $\Delta_{k}\left[r_{k}^{j} Y_{j, m}\left(\Omega_{k}\right)\right]=0$.

The scalar product in Eq. (18) can be made by use of the gradient formula. A derivation of this formula can be found for instance in Chapter 5 of Ref. [22], from which one has

$$
\begin{aligned}
& \nabla\left[\phi(r) Y_{\ell m}\left(\Omega_{r}\right)\right] \\
& =-\left(\frac{\ell+1}{2 \ell+1}\right)^{1 / 2}\left(\frac{d}{d r}-\frac{\ell}{r}\right) \phi(r) \boldsymbol{Y}_{\ell, \ell+1, m}(\Omega) \\
& \quad+\left(\frac{\ell}{2 \ell+1}\right)^{1 / 2}\left(\frac{d}{d r}+\frac{\ell+1}{r}\right) \phi(r) \boldsymbol{Y}_{\ell, \ell-1, m}(\Omega),
\end{aligned}
$$

where

$$
\boldsymbol{Y}_{j, \ell, m}(\Omega)=\sum_{m, q} Y_{\ell, m}(\Omega)\langle\ell, m ; 1, q \mid j, m\rangle \boldsymbol{e}_{q}
$$

with $\boldsymbol{e}_{0}=\boldsymbol{e}_{z}$ and $\boldsymbol{e}_{ \pm 1}=\mp\left(\boldsymbol{e}_{x} \pm i \boldsymbol{e}_{y}\right) / \sqrt{2}$.

Use of this expression permits us to rewrite Eq. (18) as

$$
\begin{aligned}
& {\left[\left[O_{\mu}^{\lambda \dagger}, T\right], O_{\mu}^{\lambda}\right]} \\
& =\frac{\hbar^{2}}{m_{N}} \sum_{i, k=1}^{3}\left(c_{i}\right)^{\lambda}\left(c_{k}\right)^{\lambda} z_{i} z_{k} \sum_{\ell=1}^{\lambda} \sum_{m, n} \sqrt{2 \lambda+1} \\
& \times\left(\begin{array}{ccc}
\ell & \lambda-\ell & \lambda \\
m & n & -\mu
\end{array}\right) \sqrt{\frac{4 \pi(2 \lambda+1) !}{(2 \ell+1) !(2 \lambda-2 \ell+1) !}} \\
& \times x_{k}^{\lambda-\ell} Y_{\lambda-\ell, n}\left(\Omega_{x_{k}}\right)\left(\sin \varphi_{i k}\right)^{\lambda-\ell}\left(\cos \varphi_{i k}\right)^{l} y_{k}^{\lambda+\ell-2} \\
& \times(2 \lambda+1)(2 \ell+1) \sqrt{\ell \lambda} \sum_{q \eta v}(-1)^{q} Y_{\lambda-1, \eta}\left(\Omega_{y_{k}}\right) \\
& \times Y_{\ell-1, \nu}\left(\Omega_{y_{k}}\right)(-1)^{\lambda-\mu+\ell+m}\left(\begin{array}{ccc}
\lambda-1 & 1 & \lambda \\
\eta & q & \mu
\end{array}\right) \\
& \times\left(\begin{array}{lcc}
\ell-1 & 1 & \ell \\
v & -q & -m
\end{array}\right) .
\end{aligned}
$$

Writing now the two spherical harmonics in terms of a single one, and summing up three of the $3-j$ symbols [16], one gets

$$
\begin{aligned}
& {\left[\left[O_{\mu}^{\lambda \dagger}, T\right], O_{\mu}^{\lambda}\right]} \\
& =\frac{\hbar^{2}}{m_{N}} \sum_{i, k=1}^{3}\left(c_{i}\right)^{\lambda}\left(c_{k}\right)^{\lambda} z_{i} z_{k} \sum_{\ell=1}^{\lambda} \sum_{m, n} \sqrt{2 \lambda+1} \\
& \quad \times\left(\begin{array}{ccc}
\ell & \lambda-\ell & \lambda \\
m & n & -\mu
\end{array}\right) \sqrt{\frac{(2 \lambda+1) !}{(2 \ell+1) !(2 \lambda-2 \ell+1) !}} x_{k}^{\lambda-\ell} \\
& \quad \times Y_{\lambda-\ell, n}\left(\Omega_{x_{k}}\right)\left(\sin \varphi_{i k}\right)^{\lambda-\ell}\left(\cos \varphi_{i k}\right)^{l} \sqrt{\ell}(2 \ell+1) \\
& \quad \times \sqrt{\lambda}(2 \lambda+1) y_{k}^{\lambda+\ell-2} \sum_{\Lambda}(-1)^{1-\mu+m-n}
\end{aligned}
$$




$$
\begin{aligned}
& \times \sqrt{(2 \lambda-1)(2 \ell-1)(2 \Lambda+1)} Y_{\Lambda, n}^{*}\left(\Omega_{y_{k}}\right) \\
& \times\left(\begin{array}{ccc}
\lambda-1 & \ell-1 & \Lambda \\
0 & 0 & 0
\end{array}\right)\left(\begin{array}{ccc}
\lambda & \ell & \Lambda \\
\mu-m-n
\end{array}\right) \\
& \times\left\{\begin{array}{lll}
\lambda & \ell & \Lambda \\
\ell-1 & \lambda-1 & 1
\end{array}\right\}
\end{aligned}
$$

which, after summation over $m$ and $\mu$ [with the summation over $\mu$ coming from Eq. (14)], leads to Eq. (19) for the contribution of the kinetic energy operator to the energyweighted sum rule $S_{1}^{(T)}$.

We close the appendix by giving analytical expressions for the two matrix elements entering in Eq. (31) for the particular case of particles without spin. The expressions are obtained by performing analytically the integrals over $\Omega_{x_{i}}$ and $\Omega_{y_{i}}$ :

$$
\begin{aligned}
& \left\langle\mathcal{Y}_{\ell_{x_{i}} \ell_{y_{i}}}^{K L_{0} M_{0}}\left(\Omega_{i}\right)\left|O_{\mu}^{\lambda \dagger}\right| \mathcal{Y}_{\ell_{x_{i}}^{\prime \prime} \ell_{y_{i}}^{\prime \prime}}^{L^{\prime \prime} M^{\prime \prime}}\left(\Omega_{i}\right)\right\rangle \\
& =(-1)^{\mu+L_{0}+M_{0}+\ell_{y_{i}}^{\prime \prime}-\ell_{x_{i}}^{\prime \prime}} \sqrt{\frac{\left(2 L^{\prime \prime}+1\right)\left(2 L_{0}+1\right)\left(2 \ell_{x_{i}}^{\prime \prime}+1\right)\left(2 \ell_{x_{i}}+1\right)\left(2 \ell_{y_{i}}^{\prime \prime}+1\right)\left(2 \ell_{y_{i}}+1\right)}{4 \pi}}\left(\begin{array}{ccc}
L^{\prime \prime} & L_{0} & \lambda \\
-M^{\prime \prime} & M_{0} & \mu
\end{array}\right) N_{K^{\prime \prime}}^{\ell_{x_{i}}^{\prime \prime} \ell_{y_{i}}^{\prime \prime}} N_{K}^{\ell_{x_{i}} \ell_{y_{i}}}
\end{aligned}
$$

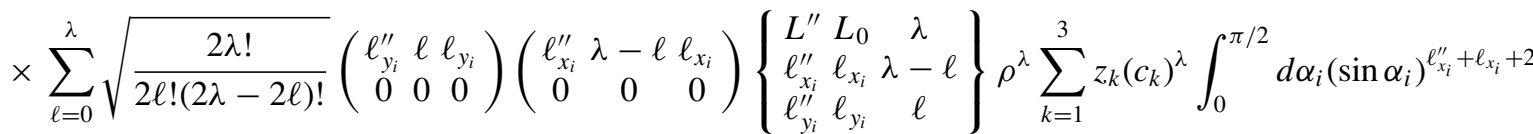

$$
\begin{aligned}
& \times\left(\cos \alpha_{i}\right)^{\ell_{y_{i}}^{\prime \prime}+\ell_{y_{i}}+2} P_{v_{i}^{\prime \prime}}^{\left(\ell_{x_{i}}^{\prime \prime}+\frac{1}{2}, \ell_{y_{i}}^{\prime \prime}+\frac{1}{2}\right)}\left(\cos 2 \alpha_{i}\right) P_{\nu_{i}}^{\left(\ell_{x_{i}}+\frac{1}{2}, \ell_{y_{i}}+\frac{1}{2}\right)}\left(\cos 2 \alpha_{i}\right)\left(\sin \varphi_{k i}\right)^{\lambda-\ell}\left(\cos \varphi_{k i}\right)^{\ell}\left(\sin \alpha_{i}\right)^{\lambda-\ell}\left(\cos \alpha_{i}\right)^{\ell} \text {, }
\end{aligned}
$$

where $N_{K}^{\ell_{x} \ell_{y}}$ is the normalization constant of the hyperspherical harmonic $\mathcal{Y}_{\ell_{x} \ell_{y}}^{K L M}(\Omega)$, whose precise form can be found for instance in Ref. [13].

[1] A. de Shalit and H. Feshbach, Theoretical Nuclear Physics (Wiley, New York, 1974).

[2] A. Bohr and B. R. Mottelson, Nuclear Structure, Vol II (Benjamin, Reading, MA, 1975).

[3] O. Bohigas, A. M. Lane, and J. Martorell, Phys. Rep. 51, 267 (1979).

[4] E. Lipparini and S. Stringari, Phys. Rep. 175, 103 (1989).

[5] A. S. Jensen, K. Riisager, D. V. Fedorov, and E. Garrido, Rev. Mod. Phys. 76, 215 (2004).

[6] H. Sagawa and M. Honma, Phys. Lett. B251, 17 (1990).

[7] T. Suzuki, H. Sagawa, and K. Hagino, Phys. Rev. C 68, 014317 (2003).

[8] T. Myo, K. Kato, S. Aoyama, and K. Ikeda, Phys. Rev. C 63, 054313 (2001).

[9] P. Descouvemont, E. Tursunov, and D. Baye, Nucl. Phys. A765, 370 (2006).

[10] R. Alvarez-Rodriguez, E. Garrido, A. S. Jensen, D. V. Fedorov, and H. O. U. Fynbo, Eur. Phys. J. A 31, 303 (2007).

[11] Y. Kanada-Enyo, Prog. Theor. Phys. 117, 655 (2007).
The expression for the second matrix element in Eq. (31) is identical to Eq. (A6) but with the function $\left[V_{i}^{\left(\ell_{x_{i}}^{\prime \prime}\right)}\left(\rho \sin \alpha_{i}\right)-\right.$ $\left.V_{i}^{\left(\ell_{x_{i}}^{\prime}\right)}\left(\rho \sin \alpha_{i}\right)\right]$ included as a factor in the integrand, and with primes on the quantum numbers $K, \ell_{x_{i}}$, and $\ell_{y_{i}}$.
[12] H. Masui, K. Kato, and K. Ikeda, Phys. Rev. C 75, 034316 (2007).

[13] E. Nielsen, D. V. Fedorov, A. S. Jensen, and E. Garrido, Phys. Rep. 347, 373 (2001).

[14] E. Garrido, D. V. Fedorov, and A. S. Jensen, Nucl. Phys. A700, 117 (2002).

[15] E. Garrido, D. V. Fedorov, and A. S. Jensen, Nucl. Phys. A733, 85 (2004).

[16] M. Rotenberg, R. Bivins, N. Metropolis, and J. K. Wooten, The 3-j and 6-j Symbols (Technology Press, MIT, Cambridge, MA, 1959).

[17] E. Garrido and E. Moya de Guerra, Nucl. Phys. A650, 387 (1999).

[18] T. Aumann et al., Phys. Rev. C 59, 1252 (1999).

[19] B. V. Danilin, I. J. Thompson, J. S. Vaagen, and M. V. Zhukov, Nucl. Phys. A632, 383 (1998).

[20] Y. Suzuki, Nucl. Phys. A528, 395 (1991).

[21] B. V. Danilin, M. V. Zhukov, J. S. Vaagen, and J. M. Bang, Phys. Lett. B302, 129 (1993).

[22] A. R. Edmonds, Angular Momentum in Quantum Mechanics (Princeton University Press, Princeton, NJ, 1974). 